\title{
Arc Effect on Charged Ion Migration in Capillary Zone Electrophoresis Using Hybrid Finite Element and Particle-in-Cell Method*
}

\author{
Cheng-I HO** and Chen-I HUNG** \\ ** Department of Mechanical Engineering, National Cheng Kung University \\ No. 1, University Road, Tainan City 70101, Taiwan (ROC) \\ E-mail: cihung@mail.ncku.edu.tw
}

\begin{abstract}
Analytes separated by capillary zone electrophoresis are governed by ion migration. The study proposed a hybrid finite element and particle-in-cell method to simulate ion migration in the cross-channel capillary zone electrophoresis system. Different to the traditional numerical method, this method can be used to handle the migration of differently charged ions for complex geometry and boundary conditions. The research focuses on the arc effect on the migration phenomena of the positive and negative ions in the injection and separation process. The results indicate that arc effect can affect obviously the migration phenomena of analytes. In the injection process, the charged ions migrate not only faster into the separation channel but also more far away from the injection channel with increasing the corner arc radius. In the separation process, the zones of charged ions are wider and the time to completely separate is longer with increasing the corner arc radius. Consequently, as corner arc radius is larger, more time is needed to inject and separate charged ions. These findings have significant implications for design and control of capillary zone electrophoresis systems.
\end{abstract}

Key words: Arc Effect, Capillary Zone Electrophoresis, Finite Element, Ion Migration, Particle-in-Cell

\section{Introduction}

Capillary zone electrophoresis (CZE) is a high efficiency analytical method to separate analytes based on their relative charge and size. The design and operation control of CZE systems have drawn great attention since the introduction of the micro total analysis system ( $\mu$ TAS) concept by Manz et al. ${ }^{(1)}$ in 1990. In 1992, Harrison et al. ${ }^{(2)}$ was the first to present CZE and sample handling system integrated on a planar glass chip. Then, the Harrison group $^{(3-6)}$ presented a series of improvements in the instrumentation and experimental method for the device. Subsequently, the significance of CZE systems has grown further with the numerous experimental studies ${ }^{(7-13)}$ in diverse application areas, including chemical, biological and medical analysis. Based on the above experimental studies, the different designs of CZE systems and the precise control strategies were developed. However, the fabrication processes for rapid prototyping and testing of new CZE system designs are slow and expensive. Considering the difficulties associated with performing experiments, analytical and numerical solutions can serve to reduce both the time and cost for development of CZE systems.

The analytical studies of electroosmotic flow (EOF) in capillaries were studied by Burgreen and Nakache ${ }^{(14)}$ and Rice and Whitehead ${ }^{(15)}$. A one-dimensional model of the CZE system was first present by Jorgenson and Lukacs ${ }^{(16)}$ in 1981. Andreev and Lisin ${ }^{(17)}$ 
investigated the effect of EOF profile on the separation efficiency in the CZE system by a one-dimensional mathematical model consisting of nonlinear partial differential equations. Dutta and Beskok ${ }^{(18)}$ proposed two-dimensional analytical solutions of mixed electroosmotic/pressure driven flows in straight microchannels. In the past decade, analytical solutions for electrokinetic transport at the nano/micrometer scale have been developed in many studies ${ }^{(19-21)}$. Due to the complexity and nonlinearities of coupled phenomena, it is difficult to obtain analytical solutions except for certain simple cases. Therefore, numerical simulations are required to aid in the design, control and optimization of CZE systems.

Patankar and $\mathrm{Hu}^{(22)}$ performed a three-dimensional simulation of EOF in a cross-channel CZE system during the injection process based on a finite volume method. Ermakov et al. ${ }^{(23,24)}$ developed a two-dimensional numerical scheme to simulate electrokinetically driven mass transport in microfabricated channel structures by employing a finite-difference algorithm. Bianchi et al. ${ }^{(25)}$ applied a finite element method (FEM) to simulating two-dimensional EOF in microscale T-junctions. Subsequently, there has been a growing interest in numerical simulation of electrokinetic transport in microchannels ${ }^{(26-34)}$.

Traditional numerical models for describing the solute concentration distribution in CZE systems are generally by solving the concentration diffusion equation. However, the migration of differently charged analytes in CZE system cannot be characterized. Therefore, particle-in-cell (PIC) method, used to solve the trajectories of charged particles on a fixed mesh in plasma physics, is adopted in this study and well-documented in the literatures ${ }^{(35-37)}$. Using finite different method is difficult to achieve the purpose of getting accurate results for complex geometry and boundary conditions in CZE systems, so this study applies a FEM to solving the governing equations. Consequently, the hybrid FEM and PIC method is used to discuss the arc effect on the migration and separation of the differently charged ions in this study. The numerical results provide useful insights into the development of CZE systems.

\section{Nomenclature}

$A$

$A_{i}$

$\mathbf{E}_{i}$

$\mathbf{E}_{\text {ion }}$

$e$

$\mathrm{H}$

h

i

j

$\mathrm{L}_{\mathrm{D}}$

$l_{\mathrm{D}}$

n

$\mathbf{P}_{\mathrm{s}}$

$p$

$q_{\text {ion }}$

Re

$\mathrm{R}_{\text {arc }}$

rarc

rion

$\mathrm{r}_{\mathrm{Ph}_{4} \mathrm{~B}^{-}}$

$\mathrm{r}_{\mathrm{Ph}_{4} \mathrm{P}^{+}}$

$t$
Total area of element enclosed by the nodal points 1,2 , and $3\left[\mathrm{~m}^{2}\right]$

Portion of shaded area within the element $\left[\mathrm{m}^{2}\right]$

Electric field intensity at the nodal point $i\left[\mathrm{~kg} \mathrm{~m} \mathrm{~s}^{-3} \mathrm{~A}^{-1}\right]$

Electric field intensity acting on the charged ion $\left[\mathrm{kg} \mathrm{m} \mathrm{s}^{-3} \mathrm{~A}^{-1}\right]$

Elementary charge [s A]

Length of the injection channel between reservoirs 1 and 2 [m]

Width of the injection and separation channels [m]

Unit vector in $\mathrm{x}$-direction

Unit vector in $\mathrm{y}$-direction

Dimensionless length between injection channel and detection point

Length between injection channel and detection point [m]

Dimensionless normal direction to the wall

Dimensionless position of the charged ion within the solvent

Dimensionless pressure

Charge of the charged ion [ $\mathrm{s} \mathrm{A} \mathrm{m}^{-1}$ ]

Reynolds number

Dimensionless corner arc radius of two cross microchannels

Corner arc radius of two cross microchannels [m]

Stokes radius of the charged ion [m]

Stokes radius of $\mathrm{Ph}_{4} \mathrm{~B}^{-}[\mathrm{m}]$

Stokes radius of $\mathrm{Ph}_{4} \mathrm{P}^{+}[\mathrm{m}]$

Dimensionless time 
$\mathrm{u}_{\mathrm{EOF}} \quad$ Dimensionless velocity component of the EOF in x-direction

$\mathbf{V}_{\text {EOF }} \quad$ Dimensionless velocity of the EOF

$\mathbf{V}_{\text {ion }}$

Dimensionless velocity of the charged ion

$\mathbf{V}_{\mathrm{s}}$

Dimensionless velocity of the charged ion within the solvent

VEOF

Dimensionless velocity component of the EOF in y-direction

W

Length of the separation channel between reservoirs 3 and 4 [m]

$\mathrm{X}, \mathrm{Y}$

Dimensionless coordinate system

$\mathrm{x}, \mathrm{y}$

Coordinate system in Fig. 1 [m]

$Z_{\text {ion }}$

Charge number of the charged ion

$Z_{\mathrm{Ph}_{4} \mathrm{~B}^{-}}$

Charge number of $\mathrm{Ph}_{4} \mathrm{~B}^{-}$

$Z_{\mathrm{Ph}_{4} \mathrm{P}^{+}}$

Charge number of $\mathrm{Ph}_{4} \mathrm{P}^{+}$

\section{Greek letters}

$\Delta \mathrm{t}$

$\varepsilon$

$\zeta$

$\eta$

$\kappa^{-1}$

$(\kappa h)^{-1}$

$\rho_{e i}$

$\rho$

$\phi$

$\varphi$

$\psi$

\section{Subscripts}

arc

D

EOF

$e i$

$i$

in

ion

$\mathrm{s}$

se

W

\section{Dimensionless time step}

Electrical permittivity of the solvent $\left[\mathrm{s}^{4} \mathrm{~A}^{2} \mathrm{~kg}^{-1} \mathrm{~m}^{-3}\right]$

Zeta potential [ $\mathrm{kg} \mathrm{m}^{2} \mathrm{~s}^{-3} \mathrm{~A}^{-1}$ ]

Dynamic viscosity of the solvent $\left[\mathrm{kg} \mathrm{m}^{-1} \mathrm{~s}^{-1}\right]$

Debye length [m]

Dimensionless Debye length

Electric charge density of the ion acting on the nodal point $i\left[\mathrm{~s} \mathrm{~A} \mathrm{~m}^{-3}\right]$

Density of the solvent $\left[\mathrm{kg} \mathrm{m}^{-3}\right]$

Dimensionless electric potential induced by external voltage

External voltage $\left[\mathrm{kg} \mathrm{m}^{2} \mathrm{~s}^{-3} \mathrm{~A}^{-1}\right.$ ]

Dimensionless electric potential induced by the charges on the wall

\section{Corner arc}

Detection point

Electroosmotic flow

Charged ion acting on the nodal point

Nodal point

Injection

Charged ion

Charged ion within the solvent

Separation

Wall

\section{Mathematical Model}

The geometry of the cross-channel CZE system consists of two cross microchannels and four reservoirs numbered 1-4 in this study, as shown schematically in Fig. 1. In the injection process, the EOF of the solvent is driven from reservoir 1 to reservoir 2 by applying an electric potential between reservoirs 1 and 2. In the subsequent separation process, the EOF of the solvent is driven from reservoir 3 to reservoir 4 by applying an electric potential between reservoirs 3 and 4. In Fig. 1, rarc denotes the corner arc radius of two cross microchannels and $l_{\mathrm{D}}$ denotes the length between injection channel and detection point in the cross-channel CZE system.

The distribution of the electric potential can be decomposed into a potential due to the external electric field and a potential due to the charges on the wall. The dimensionless electric potential $\phi$ induced by the external voltage $\varphi$ is calculated by

$$
\nabla^{2} \phi=-\left(\frac{\mathrm{h}^{2}}{\varphi}\right) \frac{\rho_{e i}}{\varepsilon} .
$$

The boundary conditions in the injection process are given by 


$$
\begin{aligned}
& \phi_{1}=1, \quad \phi_{2}=0, \phi_{3}=\phi_{4}=0.5, \\
& \frac{\partial \phi_{\mathrm{w}}}{\partial \mathrm{n}}=0 .
\end{aligned}
$$

In the separation process, the Eq. (2) is substituted by $\phi_{1}=\phi_{2}=0.5, \phi_{3}=1, \phi_{4}=0$.

\section{Injection Channel}

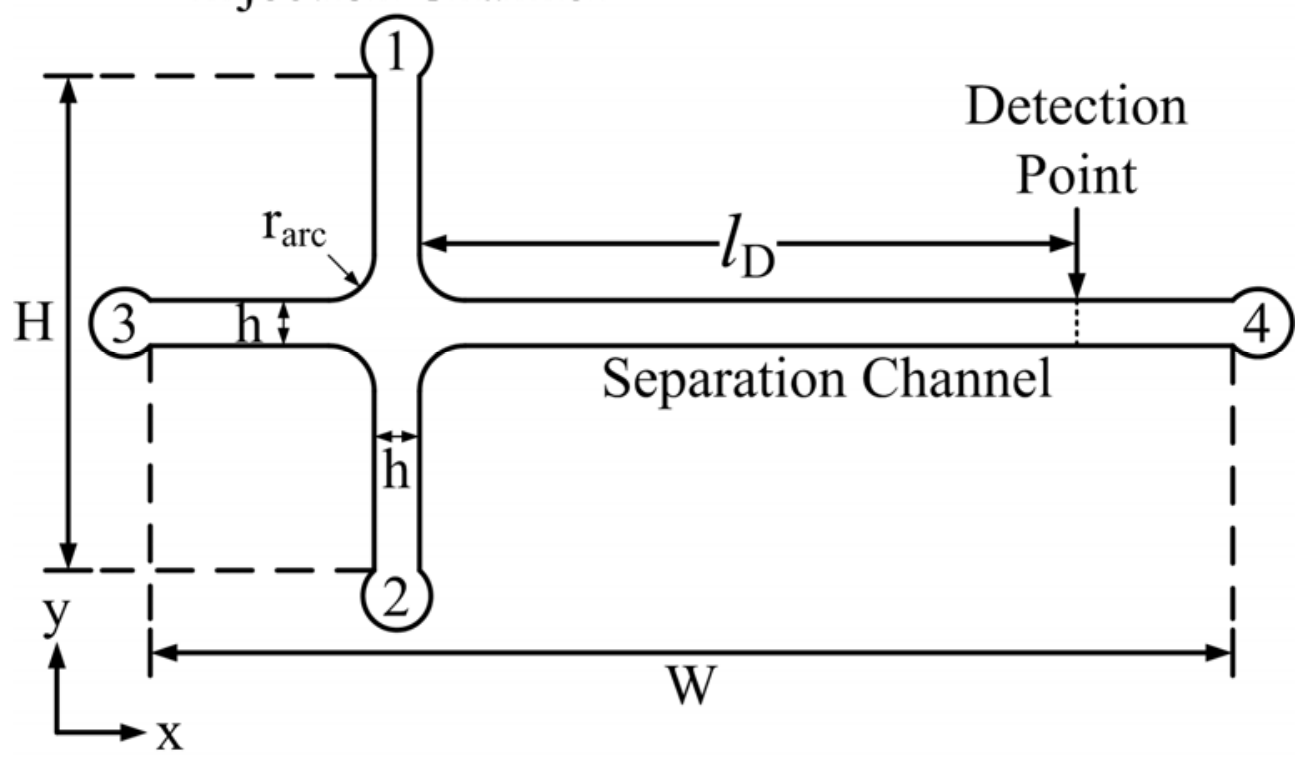

Fig. 1 Schematic illustration of the cross-channel CZE system.

Using the Debye-Hückel linear approximation ${ }^{(22)}$, the dimensionless electric potential $\psi$ induced by the charges on the wall is obtained by

$\nabla^{2} \psi=(\kappa h)^{2} \psi$.

The boundary conditions are given by

$$
\begin{aligned}
& \frac{\partial \psi_{1}}{\partial \mathrm{Y}}=0, \frac{\partial \psi_{2}}{\partial \mathrm{Y}}=0, \frac{\partial \psi_{3}}{\partial \mathrm{X}}=0, \frac{\partial \psi_{4}}{\partial \mathrm{X}}=0, \\
& \psi_{\mathrm{w}}=-1 .
\end{aligned}
$$

The dimensionless continuity and momentum equations of the incompressible $\mathrm{EOF}^{(22)}$ are given by

$$
\begin{aligned}
& \nabla \cdot \mathbf{V}_{\mathrm{EOF}}=0, \\
& \operatorname{Re}\left[\frac{\partial \mathbf{V}_{\mathrm{EOF}}}{\partial \mathrm{t}}+\left(\mathbf{V}_{\mathrm{EOF}} \cdot \nabla\right) \mathbf{V}_{\mathrm{EOF}}\right]=-\nabla p+\nabla^{2} \mathbf{V}_{\mathrm{EOF}}+\left(\kappa^{2} \mathrm{hH}\right) \psi \nabla \phi,
\end{aligned}
$$
where

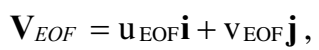

and

$$
\mathrm{Re}=\rho\left(\frac{\varphi \varepsilon \zeta}{\eta \mathrm{H}}\right)\left(\frac{\mathrm{h}}{\eta}\right) .
$$

The boundary conditions are given by

$$
\begin{aligned}
& \mathrm{u}_{\mathrm{EOF}, 1}=0, \frac{\partial \mathrm{v}_{\mathrm{EOF}, 1}}{\partial \mathrm{Y}}=0, \quad p_{\mathrm{EOF}, 1}=0 \text {, } \\
& \mathrm{u}_{\mathrm{EOF}, 2}=0, \frac{\partial \mathrm{v}_{\mathrm{EOF}, 2}}{\partial \mathrm{Y}}=0, \quad p_{\mathrm{EOF}, 2}=0, \\
& \frac{\partial \mathrm{u}_{\mathrm{EOF}, 3}}{\partial \mathrm{X}}=0, \quad \mathrm{v}_{\mathrm{EOF}, 3}=0, \quad p_{\mathrm{EOF}, 3}=0,
\end{aligned}
$$




$$
\begin{aligned}
& \frac{\partial \mathrm{u}_{\mathrm{EOF}, 4}}{\partial \mathrm{X}}=0, \quad \mathrm{~V}_{\mathrm{EOF}, 4}=0, \quad p_{\mathrm{EOF}, 4}=0, \\
& \mathrm{u}_{\mathrm{w}}=0, \quad \mathrm{v}_{\mathrm{w}}=0, \frac{\partial p_{\mathrm{w}}}{\partial \mathrm{n}}=0 .
\end{aligned}
$$

These dimensionless governing equations are derived by scaling the length by the channel width $\mathrm{h}$, the electric potential $\phi$ by the external voltage $\varphi$, the electric potential $\psi$ by the zeta potential $\zeta$, the velocity $\mathbf{V}_{E O F}$ by $\varphi \varepsilon \zeta / \eta \mathrm{H}$, and the pressure $p$ by $\varphi \varepsilon \zeta / \mathrm{hH}$.

\section{Numerical Scheme}

In this study, an algorithm based on FEM using linear triangular elements is applied to solving the dimensionless governing equations. Based on a grid independent study carried out the results within $1 \%$ of each other finer mesh, the computational domain is discretized into unstructured triangular elements with 132230 triangles when $R_{\text {arc }}=0.1$. As shown in Fig. 2, the mesh is fine close to the walls and coarse towards the center of channel for capturing the influence of the electric double layer (EDL) near the walls

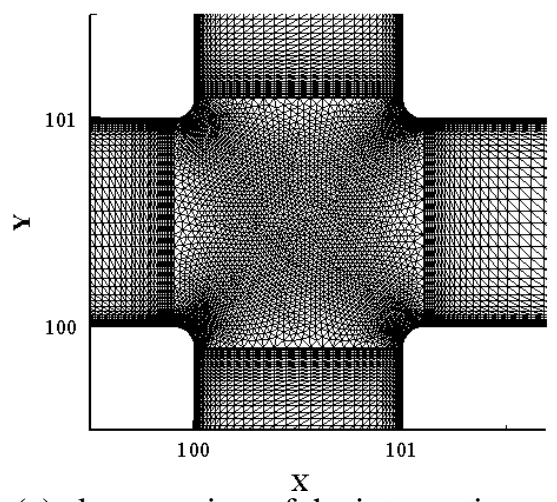

(a) close-up view of the intersection region

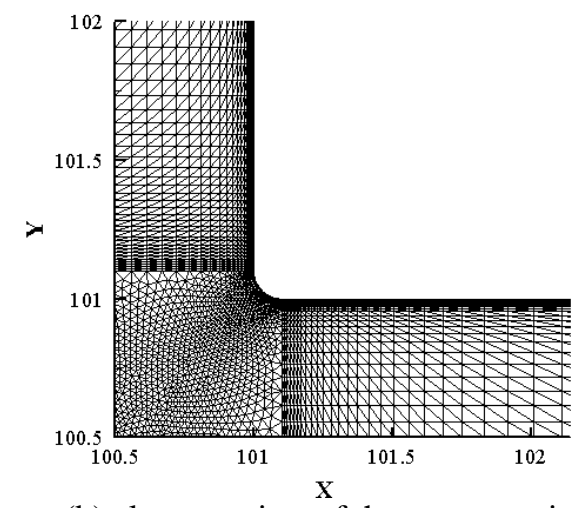

(b) close-up view of the corner region

Fig. 2 Finite element grid system applied to the cross-channel CZE system when the corner arc radius, $\mathrm{R}_{\text {arc }}=0.1$.

The PIC method is used to simulate the migration of the differently charged ions within the solvent in the cross-channel CZE system. The simulation procedure is divided into five steps and described as the following.

The first step is to compute the electric charge density of each nodal point. As shown in Fig. 3, the computational domain is discretized into three-node triangular elements. When the charged ion is located at point $\mathrm{P}$ within the triangular element enclosed by three nodal points 1,2 , and 3 , the electric charge density of the nodal point $i, \rho_{e i}$, is calculated by the portion of shaded area within the element, $A_{i}$, as the following relationship:

$$
\rho_{e i}=\frac{q_{i o n}}{A}\left(\frac{A_{i}}{A}\right), \quad i=1,2,3,
$$

where

$$
A=A_{1}+A_{2}+A_{3} \text {. }
$$

The second step is to compute the dimensionless electric field $\phi$ induced by the external voltage $\varphi$. After the electric charge density, $\rho_{e i}$, is solved in the first step, the dimensionless electric potential $\phi$ is computed by substituting $\rho_{e i}$ into Eq. (1) subject to the boundary conditions Eqs. (2) (3) in the injection process. In the separation process, the boundary condition Eq. (2) is substituted by Eq. (4). 


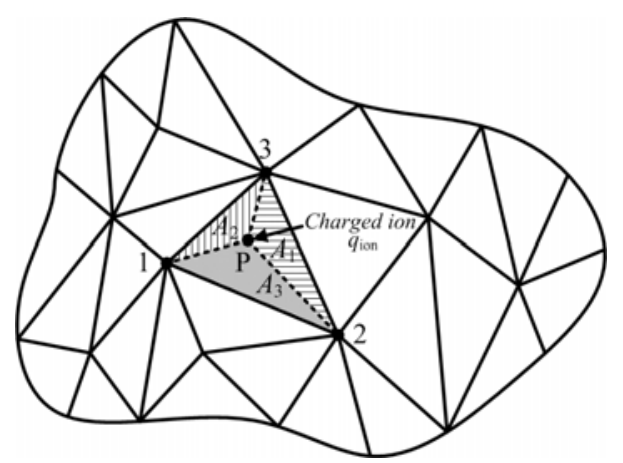

Fig. 3 Distribution of charged ion to three nodal points. The charged ion is located at point $\mathrm{P}$ within the triangular element enclosed by three nodal points 1,2 , and 3 .

The third step is to compute the dimensionless electric potential $\psi$ induced by the charges on the wall. In this step, the dimensionless electric potential $\psi$ is computed by Eq. (5) subject to the boundary conditions Eqs. (6) (7).

The fourth step is to compute the dimensionless flow field in the cross-channel CZE system. Substituting the dimensionless electric potential $\phi$ and $\psi$, respectively solved in the step 2 and 3, into Eq. (9), the dimensionless velocity of the EOF, $\mathbf{V}_{E O F}$, is computed by Eqs. (8) (9) subject to the boundary conditions Eqs. (12) (16).

The fifth step is to compute the new position of each charged ion. Due to the weak solute concentration in CZE systems, the effect of collisions between the ionic solutes can be neglected. The mobility of the ions is a key parameter governing migration of ions in CZE systems. It can be distinguished into absolute, actual, and effective mobility ${ }^{(38)}$. Experimentally, in the nonaqueous solvent, acetonitrile $(\mathrm{MeCN})$, the migration of the tetraphenylphosphonium cation $\left(\mathrm{Ph}_{4} \mathrm{P}^{+}\right)$and tetraphenylborate anion $\left(\mathrm{Ph}_{4} \mathrm{~B}^{-}\right)$is consistent with absolute mobility ${ }^{(39)}$. Based on absolute mobility ${ }^{(38)}$, the dimensionless velocity, $\mathbf{V}_{\text {ion }}$, is given by the balance between the electric field force acting on the charged ion and the retarding frictional force as the following:

$$
\mathbf{V}_{\text {ion }}=\frac{Z_{\text {ion }} e \mathbf{E}_{\text {ion }}}{6 \pi \eta r_{\text {ion }}} /\left(\frac{\varphi \varepsilon \zeta}{\eta \mathrm{H}}\right) .
$$

The electric field intensity acting on the charged ion, $\mathbf{E}_{\text {ion }}$, in Eq. (19) is distributed by the electric field intensity at nodal points 1, 2, and 3 as shown in Fig. 3. The relationship can be expressed as the following:

$$
\mathbf{E}_{\text {ion }}=\sum_{i=1}^{3} \mathbf{E}_{i}\left(\frac{A_{i}}{A}\right) .
$$

where the electric field intensity at the nodal point $i, \mathbf{E}_{i}$, is computed by the relationship in electrodynamics as the following

$$
\mathbf{E}_{i}=-\frac{1}{\mathrm{~h}} \nabla\left(\varphi \phi_{i}+\zeta \psi_{i}\right) .
$$

The new dimensionless position of the charged ion within the solvent, $\mathbf{P}_{\mathrm{s}}(\mathrm{t}+\Delta \mathrm{t})$, can be calculated by

$$
\mathbf{P}_{\mathrm{s}}(\mathrm{t}+\Delta \mathrm{t})=\mathbf{P}_{\mathrm{s}}(\mathrm{t})+\left(\mathbf{V}_{\mathrm{s}} \cdot \Delta \mathrm{t}\right),
$$

where the dimensionless velocity of the charged ion within the solvent, $\mathbf{V}_{\mathrm{s}}$, is the sum of the dimensionless velocity of the EOF, $\mathbf{V}_{\mathrm{EOF}}$, and the dimensionless ion velocity, $\mathbf{V}_{\text {ion }}$, as the following:

$$
\mathbf{V}_{\mathrm{s}}=\mathbf{V}_{\mathrm{EOF}}+\mathbf{V}_{\text {ion }},
$$

and the dimensionless time step $\Delta \mathrm{t}$ is determined by ${ }^{(22)}$

$$
\Delta \mathrm{t}=0.467 \mathrm{Re} \text {. }
$$

Once the new dimensionless position of the each charged ion within the solvent, $\mathbf{P}_{\mathrm{s}}(\mathrm{t}+\Delta \mathrm{t})$ is solved, the simulation procedure from step 1 to 5 is completed in this 
dimensionless time step. For the next dimensionless time step, the procedure immediately returns to step 1 and is repeated iteratively to obtain the migration of all the ionic solutes in the cross-channel CZE system.

In this study, the ionic solutes, $\mathrm{Ph}_{4} \mathrm{P}^{+}$and $\mathrm{Ph}_{4} \mathrm{~B}^{-}$, and a buffer solvent, MeCN, with physical properties $^{(39)}$ listed in Table 1 is adopted. Calculating Eq. (11) by substituting the values listed in Table 1, the Reynolds number is obtained to be 0.53 .

Table 1 Physical properties and parameters of the cross-channel CZE system

\begin{tabular}{lll}
\hline Parameters & Value & Units \\
\hline$e$ & $1.602 \times 10^{-19}$ & $\mathrm{~s}-\mathrm{A}$ \\
$\mathrm{H}$ & $8.04 \times 10^{-3}$ & $\mathrm{~m}$ \\
$\mathrm{~h}$ & $4 \times 10^{-5}$ & $\mathrm{~m}$ \\
$\mathrm{r}_{\text {arc }}$ & $0,4 \times 10^{-6}, 4 \times 10^{-5}$ & $\mathrm{~m}$ \\
$\mathrm{r}_{\mathrm{Ph}_{4} \mathrm{~B}^{-}}$ & $4.07 \times 10^{-10}$ & $\mathrm{~m}$ \\
$\mathrm{r}_{\mathrm{Ph}_{4} \mathrm{P}^{+}}$ & $4.19 \times 10^{-10}$ & $\mathrm{~m}$ \\
$\mathrm{~W}$ & $3 \times 10^{-2}$ & $\mathrm{~m}$ \\
$Z_{\mathrm{Ph}_{4} \mathrm{~B}^{-}}$ & -1 & - \\
$Z_{\mathrm{Ph}_{4} \mathrm{P}^{+}}$ & 1 & - \\
$\varepsilon$ & $3.168033 \times 10^{-10}$ & $\mathrm{~s}-\mathrm{A}^{2}-\mathrm{kg}^{-1}-\mathrm{m}^{-3}$ \\
$\zeta$ & 0.1 & $\mathrm{~kg}-\mathrm{m}^{2}-\mathrm{s}^{-3}-\mathrm{A}^{-1}$ \\
$\eta$ & $3.41 \times 10^{-4}$ & $\mathrm{~kg}-\mathrm{m}^{-1}-\mathrm{s}^{-1}$ \\
$(\kappa \mathrm{h})^{-1}$ & $2.5 \times 10^{-3}$ & - \\
$\rho$ & $7.87 \times 10^{2}$ & $\mathrm{~kg}-\mathrm{m}^{-3}$ \\
$\varphi$ & 500 & $\mathrm{~kg}-\mathrm{m}^{2}-\mathrm{s}^{-3}-\mathrm{A}^{-1}$ \\
\hline
\end{tabular}

\section{Results and Discussion}

In the following sections, the arc effect on the migration of the positive and negative ions in the cross-channel CZE system has been studied in the both injection and separation process. This study discusses three cases of the corner arc radius, i.e. $R_{\text {arc }}=0, R_{\text {arc }}=0.1$ and $\mathrm{R}_{\mathrm{arc}}=1$. Furthermore, all the numerical results and variables are presented in the dimensionless form. The migration phenomena of the positive and negative ions, i.e., $\mathrm{Ph}_{4} \mathrm{P}^{+}$ and $\mathrm{Ph}_{4} \mathrm{~B}^{-}$, in the nonaqueous solvent, i.e., $\mathrm{MeCN}$, are investigated in the cross-channel CZE system by the hybrid FEM and PIC method.

\subsection{Charged ion migration in the injection process}

In the injection process, the reservoirs 1 and 2 are applied the values of electric potential 1 and 0 , respectively. At the same time, the reservoirs 3 and 4 are both applied the value of electric potential 0.5. These conditions induce the EOF of solvent to transport the differently charged ions from reservoir 1 to reservoir 2 .

For the cases of the different corner arc radii, substituting the above conditions into Eq. (1) yields the electrical potential field $\phi$ induced by external voltage in the cross-channel of the CZE system as shown in Fig. 4. The electric potential $\phi$ is linear variations in the center of the injection channel and only small variations extended into the separation channel. Hence, the velocity of the EOF decreases when the EOF flows into the separation channel. With increasing the corner arc radius, the variation of electric potential $\phi$ is smaller in the intersection region of the cross-channel CZE system. The results indicate that the velocity of the EOF in the intersection region is the smallest when $R_{\text {arc }}=1$. 


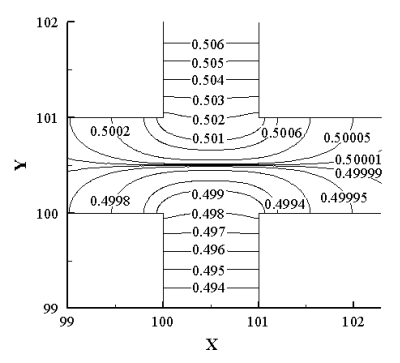

(a) $\mathrm{R}_{\text {arc }}=0$

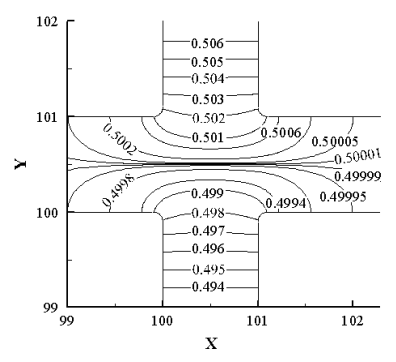

(b) $\mathrm{R}_{\text {arc }}=0.1$

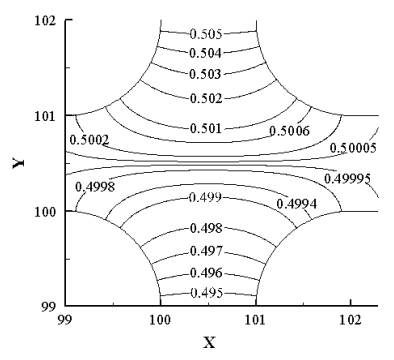

(c) $\mathrm{R}_{\text {arc }}=1$

Fig. 4 Distribution of the electric potential $\phi$ induced by external voltage in the intersection region of the cross-channel CZE system. Note $\phi=1$ at reservoir 1 , $\phi=0$ at reservoir 2, $\phi=0.5$ at reservoirs 3 and 4 .

The zeta potential on the wall is applied the values of electric potential -1 to inducing the EDL near the wall. For the cases of the different corner arc radii, Rarc, substituting the above conditions into Eq. (5) yields the electrical potential field $\psi$ induced by the charges on the wall in the cross-channel of the CZE system as shown in Fig. 5. The trend of electric potential $\psi$ is rapidly reduced to -1 near the wall. The present numerical solution is in agreement with the analytical solution ${ }^{(22)}$ when $\mathrm{R}_{\text {arc }}=0$. The decay degree of the electric potential $\psi$ is similar near the wall of the injection and separation channels for the different corner arc radii. Therefore, the impact of EDL is obvious when the charged ions migrate near the wall.

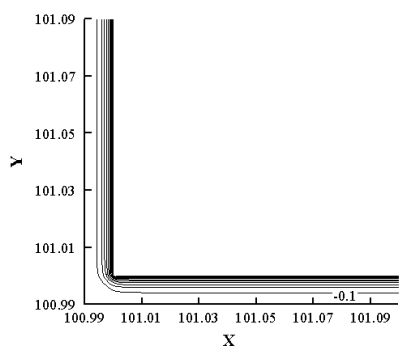

(a) $\mathrm{R}_{\text {arc }}=0$

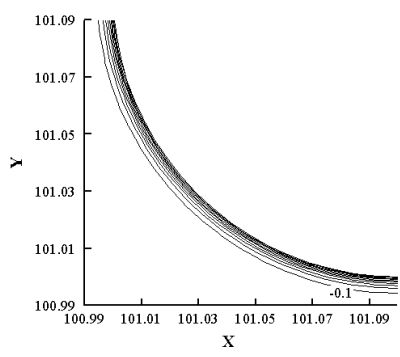

(b) $\mathrm{R}_{\mathrm{arc}}=0.1$

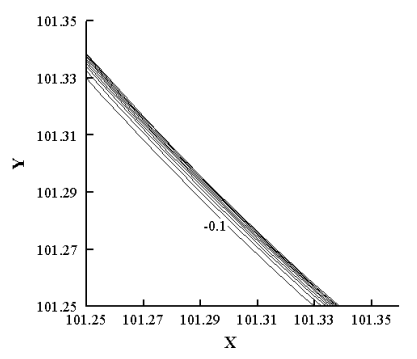

(c) $\mathrm{R}_{\mathrm{arc}}=1$

Fig. 5 Distribution of the electrical potential $\psi$ induced by the charges on the wall in the intersection region of the cross-channel CZE system. Note $\psi=-1$ at the wall.

For the cases of the different corner arc radii, the streamlines of the EOF are plotted in Fig. 6. The streamlines of the EOF which extend into the separation channel show that the EOF carries the charged ions into the separation channel. With increasing the corner arc radius, the streamlines of the EOF extend more far away from the injection channel. The results mean that the charged ions are transported more far away from the injection channel by the EOF when $\mathrm{R}_{\mathrm{arc}}=1$.

In the injecting process, the migration phenomena of the differently charged ions at the injection time $t_{\text {in }}=550$ and $t_{\text {in }}=600$ are plotted in Figs. 7 and 8 , respectively. The numerical results shown in Fig. 7(c) are consistent with the experiment study ${ }^{[9]}$. Specially, the migration phenomena of the differently charged ions can be shown clearly in this study. As shown in Eq. (23), the positive ions migrate faster than the negative ions because of the opposite sign of the ion velocity, $\mathbf{V}_{\text {ion }}$. The phenomenon can be also found in Fig. 7. Due to the effect of the EDL, the positive and negative ions are respectively attracted to and repelled away the wall when the charged ions are within the EDL. Since the charged ions are transported into the intersection region by the EOF, the positive ions migrate more far away upper wall of the separation channel and the injection channel than the negative ions. 
With increasing the corner arc radius, the positive and negative ions migrate slower and more far away the injection channel.

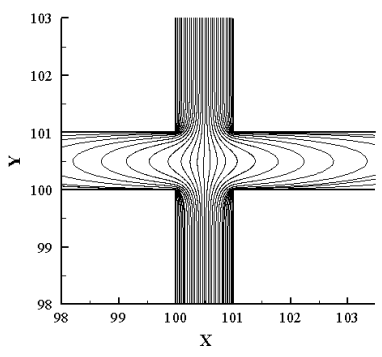

(a) $\mathrm{R}_{\text {arc }}=0$

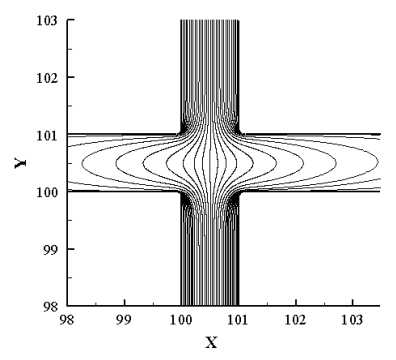

(b) $\mathrm{R}_{\text {arc }}=0.1$

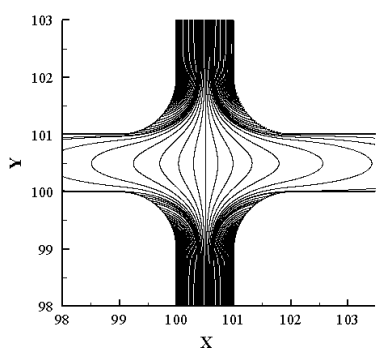

(c) $\mathrm{R}_{\mathrm{arc}}=1$

Fig. 6 Distribution of the streamlines of the EOF in the intersection region of the cross-channel CZE system. Note $\phi=1$ at reservoir $1, \phi=0$ at reservoir 2, $\phi=0.5$ at reservoirs 3 and $4, \psi=-1$ at the wall.

\section{+ Positive Ions $\Delta$ Negative Ions}

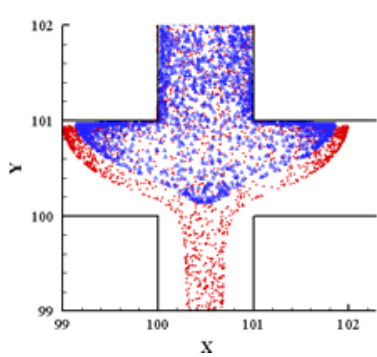

(a) $\mathrm{R}_{\text {arc }}=0$

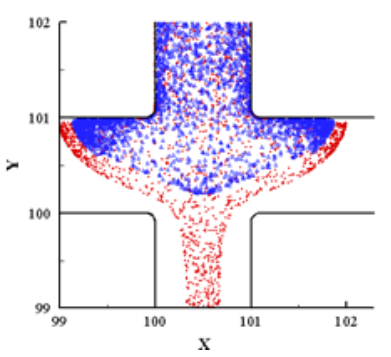

(b) $\mathrm{R}_{\text {arc }}=0.1$

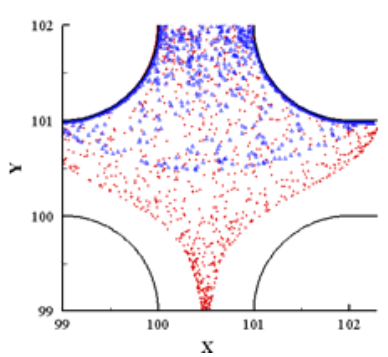

(c) $\mathrm{R}_{\text {arc }}=1$

Fig. 7 Distribution of the differently charged ions in the intersection region of the cross-channel CZE system at the injection time $t_{\text {in }}=550$.

\section{+ Positive Ions $\Delta$ Negative Ions}

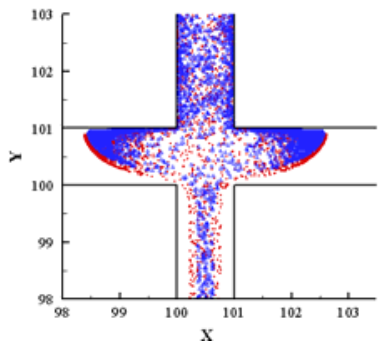

(a) $\mathrm{R}_{\text {arc }}=0$

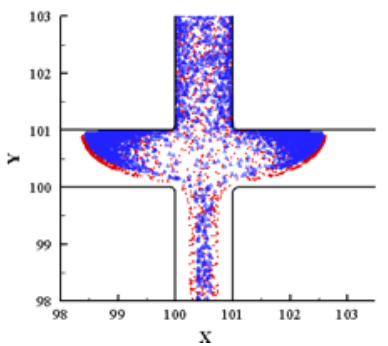

(b) $\mathrm{R}_{\mathrm{arc}}=0.1$

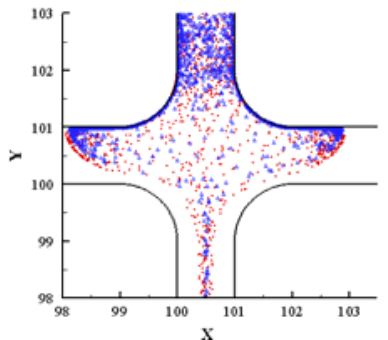

(c) $\mathrm{R}_{\text {arc }}=1$

Fig. 8 Distribution of the differently charged ions in the intersection region of the cross-channel CZE system at the injection time $t_{\text {in }}=600$.

At the injection time $t_{\text {in }}=600$, the positive and negative ions both migrate into the downstream of injection channel and accumulate in the separation channel, as shown in Fig. 8. Further, the migration of the differently charged ions has significantly differences in the separation channel because of the different corner arc radius. The positive and negative ions migrate less to the separation channel and more far away the injection channel with increasing the corner arc radius. As a result, the zones of the positive and negative ions in the separation channel are the widest when $R_{\text {arc }}=1$.

In addition, the few negative ions migrate into the separation channel at the injection time $t_{\text {in }}=550$. Therefore, the injection time $t_{\text {in }}=600$ is a convenient time to switch the electric field to subsequent separation process. 


\subsection{Charged ion migration in the separation process}

In the separation process, the reservoirs 1 and 2 are both applied the value of electric potential 0.5 , while the reservoirs 3 and 4 are applied the values of electric potential 1 and 0 , respectively. The conditions induce the EOF of solvent to transport the differently charged ions from reservoir 3 to reservoir 4.

For the cases of the different corner arc radii, substituting the above conditions into Eq. (1) yields the electrical potential field $\phi$ induced by external voltage in the cross-channel of the CZE system as shown in Fig. 9. The variation of the electric potential $\phi$ between the reservoir 3 and injection channel is larger than one between the reservoir 4 and injection channel. Also, there are only small variations extended into the injection channel. The results present that the velocity of the EOF between the reservoir 3 and injection channel is larger than one between the reservoir 4 and injection channel. With increasing the corner arc radius, the variation of electric potential $\phi$ is smaller in the intersection region of the cross-channel CZE system. The results indicate that the velocity of the EOF in the intersection region is the smallest when $R_{\text {arc }}=1$

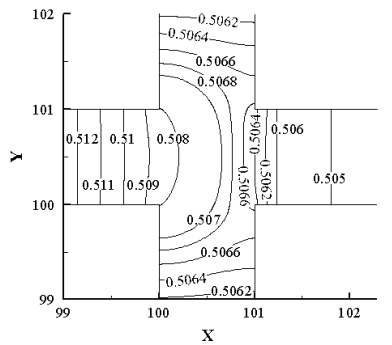

(a) $\mathrm{R}_{\text {arc }}=0$

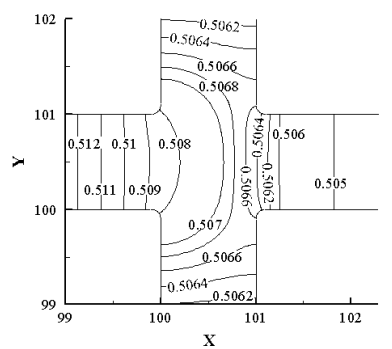

(b) $\mathrm{R}_{\text {arc }}=0.1$

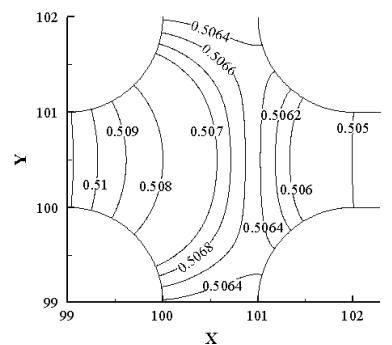

(c) $\mathrm{R}_{\text {arc }}=1$

Fig. 9 Distribution of the electric potential $\phi$ induced by external voltage in the intersection region of the cross-channel CZE system. Note $\phi=1$ at reservoir 3 , $\phi=0$ at reservoir $4, \phi=0.5$ at reservoirs 1 and 2 .

For the cases of the different corner arc radii, the streamlines of the EOF are plotted in Fig. 10. There is a little distortion of the streamlines of the EOF in the intersection region. The results mean that a few charged ions are carried into or from the injection channel. Therefore, most of the charged ions in the separation are directly carried into the downstream of separation channel by the EOF. With increasing the corner arc radius, $\mathrm{R}_{\mathrm{arc}}$, the streamlines of the EOF are more curved in the intersection region.

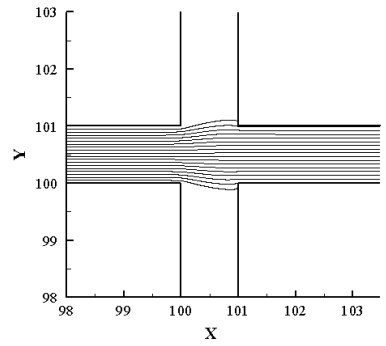

(a) $\mathrm{R}_{\text {arc }}=0$

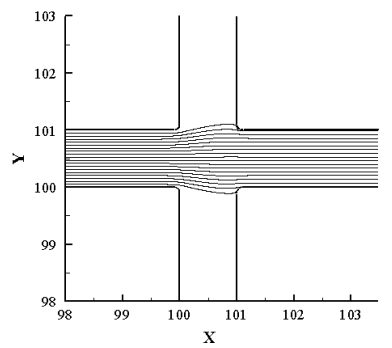

(b) $\mathrm{R}_{\mathrm{arc}}=0.1$

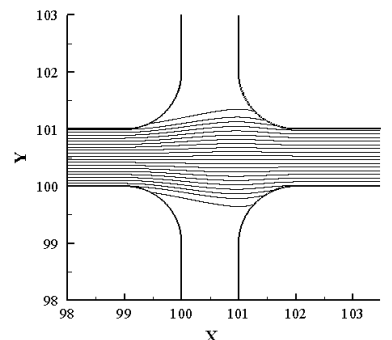

(c) $\mathrm{R}_{\text {arc }}=1$

Fig. 10 Distribution of the streamlines of the EOF in the intersection region of the cross-channel CZE system. Note $\phi=1$ at reservoir $3, \phi=0$ at reservoir 4, $\phi=0.5$ at reservoirs 1 and $2, \psi=-1$ at the wall. 
The positive ions migrate faster than the negative ions in the separation channel at the separation time $t_{\mathrm{se}}=10$, as shown in Fig. 11. Hence, the distinction between the zones of the positive and negative ions is clearly observed. With increasing the corner arc radius, the zones of the positive and negative ions are wider.

+ Positive Ions $\Delta$ Negative Ions

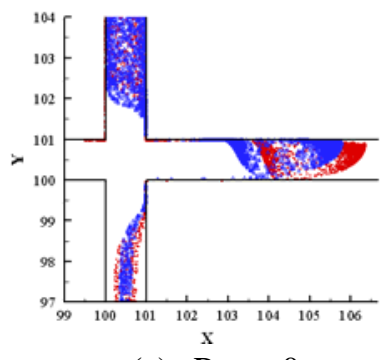

(a) $\mathrm{R}_{\text {arc }}=0$

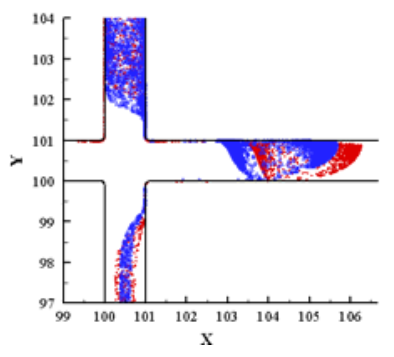

(b) $\mathrm{R}_{\text {arc }}=0.1$

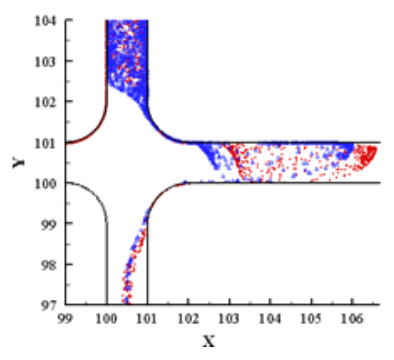

(c) $\mathrm{R}_{\text {arc }}=1$

Fig. 11 Distribution of the differently charged ions in the separation channel of the cross-channel CZE system at the separation time $t_{\text {se }}=10$.

In order to realize the migration and separation phenomena of differently charged ions specifically, Fig. 12 plots the zoom areas of the separation channel in the differently corner arc radii and separation time. Due to the EDL near the wall, the negative ions migrate more near the wall than the positive ions and slow near the wall. With increasing the separation time, the zones of the positive and negative ions both become narrower because the migration velocity is decreasing when the differently charged ions migrate away from the intersection region. With increasing corner arc radius, the zones of the positive and negative ions need more time to separate.

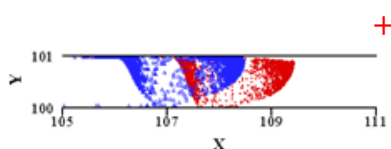

(a) $\mathrm{R}_{\text {arc }}=0, \mathrm{t}_{\mathrm{se}}=20$

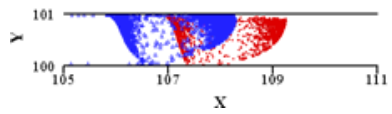

(d) $\mathrm{R}_{\mathrm{arc}}=0.1, \mathrm{t}_{\mathrm{se}}=20$

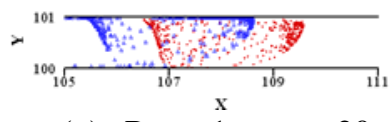

(g) $\mathrm{R}_{\text {arc }}=1, \mathrm{t}_{\mathrm{se}}=20$
+ Positive Ions

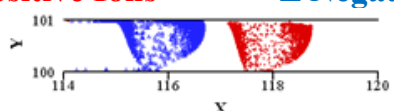

(b) $\mathrm{R}_{\mathrm{arc}}=0, \mathrm{t}_{\mathrm{se}}=60$

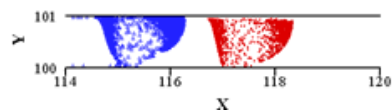

(e) $\mathrm{R}_{\text {arc }}=0.1, \mathrm{t}_{\mathrm{se}}=60$

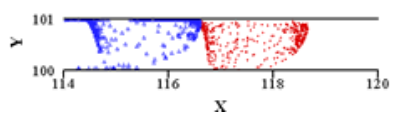

(h) $\mathrm{R}_{\mathrm{arc}}=1, \mathrm{t}_{\mathrm{se}}=60$

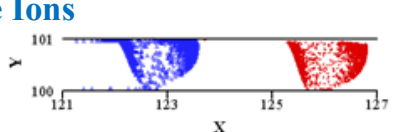

(c) $\mathrm{R}_{\mathrm{arc}}=0, \mathrm{t}_{\mathrm{se}}=100$

Fig. 12 Distribution of the differently charged ions in the zoom area of the separation channel.

Figure 13 illustrates the intensity of the positive and negative ions in the separation process. The results show that the positive and negative ions separately accumulate at the sides of the each zone. The intensity of negative ions is higher than the intensity of positive ions because of the EDL. With increasing corner arc radius, the intensity of the positive and negative ions decreases at the sides of the zone. However, the intensity of the negative ions increases at the sides of the zone when $R_{\text {arc }}=1$ because some negative ions migrate from the intersection region to the downstream of the separation channel. 


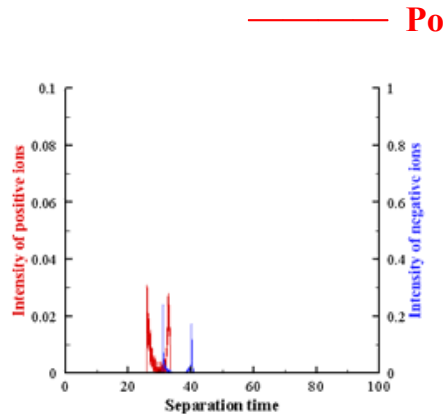

(a) $\mathrm{R}_{\mathrm{arc}}=0, \mathrm{~L}_{\mathrm{D}}=10$
Positive Ions

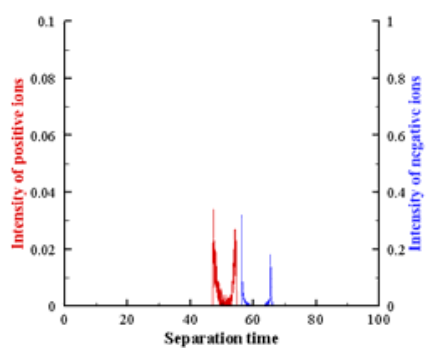

(b) $\mathrm{R}_{\mathrm{arc}}=0, \mathrm{~L}_{\mathrm{D}}=15$
Negative Ions

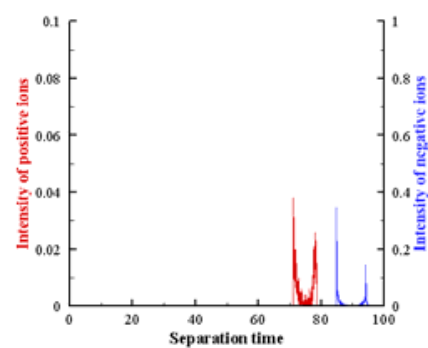

(c) $\mathrm{R}_{\mathrm{arc}}=0, \mathrm{~L}_{\mathrm{D}}=20$

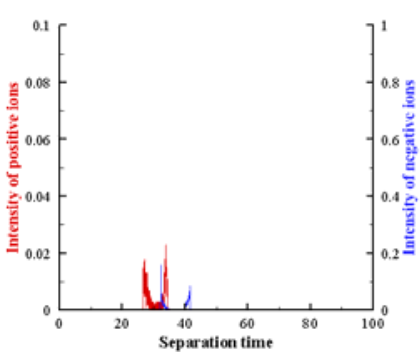

(d) $\mathrm{R}_{\text {arc }}=0.1, \mathrm{~L}_{\mathrm{D}}=10$

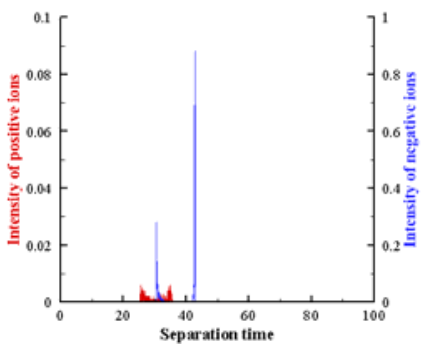

(g) $\mathrm{R}_{\mathrm{arc}}=1, \mathrm{~L}_{\mathrm{D}}=10$

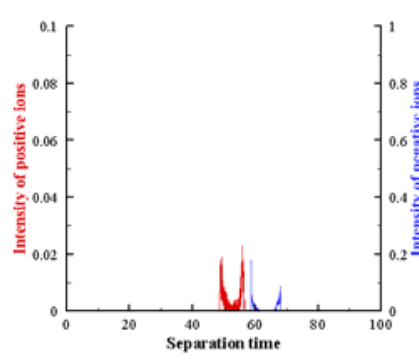

(e) $\mathrm{R}_{\text {arc }}=0.1, \mathrm{~L}_{\mathrm{D}}=15$

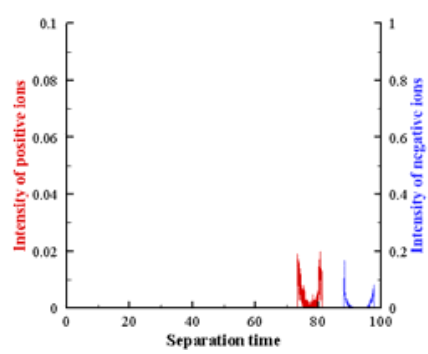

(f) $\mathrm{R}_{\mathrm{arc}}=0.1, \mathrm{~L}_{\mathrm{D}}=20$

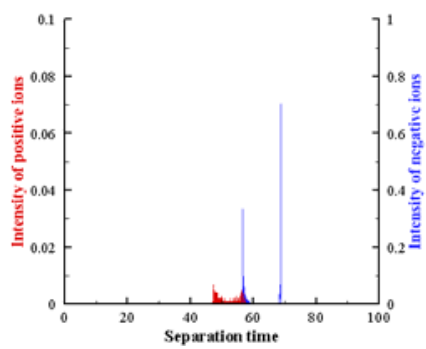

(h) $\mathrm{R}_{\text {arc }}=1, \mathrm{~L}_{\mathrm{D}}=15$

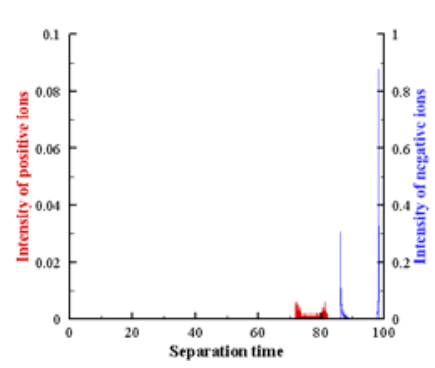

(i) $\mathrm{R}_{\mathrm{arc}}=1, \mathrm{~L}_{\mathrm{D}}=20$

Fig. 13 Intensity of the differently charged ions in the separation channel.

\section{Conclusions}

The study applies the hybrid FEM and PIC method to investigating the arc effect on the migration phenomena of the positive and negative ions in the cross-channel CZE system during the injection and separation process. The numerical results are congruent with the experimental observations. The results of this study point to several conclusions as follows:

1. The positive ions migrate not only more far away upper wall of the separation channel and the injection channel but also faster than the negative ions in the injection process.

2. The zones of positive and negative ions in the separation channel are wider and the amount of charged ions decreases with increasing the corner arc radius in the injection process.

3. The injection time $t_{\text {in }}=600$ is suitable for switching the electric field to subsequent separation process.

4. The positive and negative ions in the separation channel begin to migrate separately in group to separate into distinct zones and accumulate at the sides of the each zone in the separation process.

5. The zones of positive and negative ions are wider and need more time to separate with increasing the corner arc radius in the separation process.

6. The intensity of the positive and negative ions decreases with increasing corner arc radius and the intensity of negative ions is higher than the intensity of positive ions in the separation process.

The results provide a foundation for faster analysis and design of CZE assays. 


\section{References}

(1) Manz, A., Graber, N. and Widmer, H.M., Miniaturized Total Chemical Analysis Systems: a Novel Concept for Chemical Sensing, Sensors and Actuators B: Chemical, Vol.1, No.1-6(1990), pp.244-248.

(2) Harrison, D.J., Manz, A., Fan, Z., Lüdi, H. and Widmer, H.M., Capillary Electrophoresis and Sample Injection Systems Integrated on a Planar Glass Chip, Analytical Chemistry, Vol.64, No.17(1992), pp.1926-1932.

(3) Harrison, D.J., Glavina, P.G. and Manz, A., Towards Miniaturized Electrophoresis and Chemical Analysis Systems on Silicon: an Alternative to Chemical Sensors, Sensors and Actuators B: Chemical, Vol.10, No.2(1993), pp.107-116.

(4) Seiler, K., Harrison, D.J. and Manz, A., Planar Glass Chips for Capillary Electrophoresis: Repetitive Sample Injection, Quantitation, and Separation Efficiency, Analytical Chemistry, Vol.65, No.10(1993), pp.1481-1488.

(5) Fan, Z.H. and Harrison, D.J., Micromachining of Capillary Electrophoresis Injectors and Separators on Glass Chips and Evaluation of Flow at Capillary Intersections, Analytical Chemistry, Vol.66, No.1(1994), pp.177-184.

(6) Seiler, K., Fan, Z.H., Fluri, K. and Harrison, D.J., Electroosmotic Pumping and Valveless Control of Fluid Flow within a Manifold of Capillaries on a Glass Chip, Analytical Chemistry, Vol.66, No.20(1994), pp.3485-3491.

(7) Gong, M., Wehmeyer, K. R., Stalcup, A. M., Limbach, P. A. and Heineman, W. R., Study of Injection Bias in a Simple Hydrodynamic Injection in Microchip CE, Electrophoresis, Vol.28, No.10(2007), pp.1564-1571.

(8) Kaigala, G. V., Hoang, V. N., Stickel, A., Lauzon, J., Manage, D., Pilarski, L. M. and Backhouse, C. J., An Inexpensive and Portable Microchip-based Platform for Integrated RT-PCR and Capillary Electrophoresis, Analyst, Vol.133, No.3(2008), pp.331-338.

(9) Mühlberger, H., Hwang, W., Guber, A. E., Saile, V., and Hoffmann, W., Polymer Lab-on-a-Chip System With Electrical Detection, IEEE Sensors Journal, Vol.8, No.5(2008), pp.572-579.

(10) Yanagisawa, N. and Dutta, D., Pressure Generation at the Junction of Two Microchannels with Different Depths, Electrophoresis, Vol.31, No.12(2010), pp.2080-2088.

(11) Xia, L., and Dutta, D., A Microchip Device for Enhancing Capillary Zone Electrophoresis Using Pressure-Driven Backflow, Analytical Chemistry, Vol.84, No.22(2012), pp.10058-10063.

(12) Xia, L., and Dutta, D., Microfluidic Flow Counterbalanced Capillary Electrophoresis, Analyst, Vol.138, No.7(2013), pp.2126-2133.

(13) Segato, T. P., Bhakta, S. A., Gordon, M. T., Carrilho, E., Willis, P. A., Jiao, H., and Garcia, C. D., Microfab-less Microfluidic Capillary Electrophoresis Devices, Analytical Methods, Vol.5, No.7(2013), pp.1652-1657.

(14) Burgreen, D. and Nakache, F. R., Electrokinetic Flow in Ultrafine Capillary Slits, The Journal of Physical Chemistry, Vol.68, No.6(1964), pp.1084-1091.

(15) Rice, C.I. and Whitehead, R., Electrokinetic Flow in a Narrow Cylindrical Capillary, The Journal of Physical Chemistry, Vol.69, No.11(1965), pp.4017-4024.

(16) Jorgenson, J.W. and Lukacs, K.D., Zone Electrophoresis in Open-Tubular Glass Capillaries, Analytical Chemistry, Vol.53, No.8(1981), pp.1298-1302.

(17) Andreev, V.P. and Lisin, E.E., On The Mathematical Model of Capillary Electrophoresis, Chromatographia, Vol.37, No.3-4(1993), pp.202-210.

(18) Dutta, P. and Beskok, A., Analytical Solution of Combined Electroosmotic/Pressure Driven Flows in Two-Dimensional Straight Channels: Finite Debye Layer Effects, Analytical Chemistry, Vol.73, No.9(2001), pp.1979-1986. 
(19) Pennathur, S. and Santiago, J. G., Electrokinetic Transport in Nanochannels. 1. Theory, Analytical Chemistry, Vol.77, No.21 (2005), pp.6772-6781.

(20) Dutta, D., Electrokinetic Transport of Charged Samples through Rectangular Channels with Small Zeta Potentials, Analytical Chemistry, Vol.80, No.12(2008), pp.4723-4730.

(21) Movahed, S., Kamali, R., Eghtesad, M. and Khosravifard, A., Analytical Study of Mixed Electroosmotic-Pressure-Driven Flow in Rectangular Micro-Channels, Theoretical and Computational Fluid Dynamics, (2012). doi:10.1007/s00162-012-0283-9

(22) Patankar, N.A. and Hu, H.H., Numerical Simulation of Electroosmotic Flow, Analytical Chemistry, Vol.70, No.9(1998), pp.1870-1881.

(23) Ermakov, S.V., Jacobson, S.C. and Ramsey, J.M., Computer Simulations of Electrokinetic Transport in Microfabricated Channel Structures, Analytical Chemistry, Vol.70, No.21(1998), pp.4494-4504.

(24) Ermakov, S.V., Jacobson, S.C. and Ramsey, J.M., Computer Simulations of Electrokinetic Injection Techniques in Microfluidic Devices, Analytical Chemistry, Vol.72, No.15(2000), pp.3512-3517.

(25) Bianchi, F., Ferrigno, R. and Girault, H.H., Finite Element Simulation of an Electroosmotic-Driven Flow Division at a T-Junction of Microscale Dimensions, Analytical Chemistry, Vol.72, No.9(2000), pp.1987-1993.

(26) Ren, L., Sintonm, D. and Li, D., Numerical Simulation of Microfluidic Injection Processes in Crossing Microchannels, Journal of Micromechanics and Microengineering, Vol.13, No.5(2003), pp.739-747.

(27) Hsu, M. Y. and Hung, C. I., Simulation of Charged Ion Migration in Capillary Zone Electrophoresis System using Particle in Cell Method, Japanese Journal of Applied Physics, Vol.46, No.6A(2007), pp.3605-3612.

(28) Hsu, M. Y. and Hung, C. I., Simulation of Absolute Mobility Ionic Migration in Non-Aqueous Capillary Zone Electrophoresis, International Journal of Transport Phenomena, Vol.11, No.1(2009), pp.19-38.

(29) Kler, P. A., López, E. J., Dalcín, L. D., Guarnieri, F. A. and Storti, M. A., High Performance Simulations of Electrokinetic Flow and Transport in Microfluidic Chips, Computer Methods in Applied Mechanics and Engineering, Vol.198, No.30-32(2009), pp.2360-2367.

(30) Barz, D. P. J., Comprehensive Model of Electrokinetic Flow and Migration in Microchannels with Conductivity Gradients, Microfluidics and Nanofluidics, Vol.7, No.2(2009), pp.249-265.

(31) Prachittham, V., Picasso, M. and Gijs, M. A. M., Adaptive Finite Elements with Large Aspect Ratio for Mass Transport in Electroosmosis and Pressure-Driven Microflows, International Journal for Numerical Methods in Fluids, Vol.63, No.9(2010), pp.1005-1030.

(32) Lee, D. H., Farouk, B., and Noh, H., 3-D Simulations of Electroosmotic Sample Migration in Microchannels: Effects of Surface and Solution Property Variations, Separation Science and Technology, Vol.46, No.9(2011), pp.1337-1387.

(33) Bera, S. and Bhattacharyya, S., On Mixed Electroosmotic-Pressure Driven Flow and Mass Transport in Microchannels, International Journal of Engineering Science, Vol.62(2013), pp.165-176.

(34) Charhrouchni, I., Pallandre, A., Le Potier, I., Deslouis, C. and Haghiri-Gosnet, A. M., Computational Study of Velocity Profile Obtained in Microfluidic Channel Bearing a Fluidic Transistor: Toward Highly Resolved Electrophoretic Separation, Electrophoresis, Vol.34, No.5(2013), pp.725-735. 
(35) Birdsall, C.K. and Langdon, A.B., Plasma Physics via Computer Simulation, (1985), McGraw-Hill.

(36) Hockney, R.W. and Eastwood, J.W., Computer Simulation Using Particles, (1988), Institute of Physics Publishing.

(37) Birdsall, C.K., Particle-in-Cell Charged-Particle Simulations, Plus Monte Carlo Collisions with Neutral Atoms, PIC-MCC, IEEE Transactions on Plasma Science, Vol.19, No.2(1991), pp.65-85.

(38) Porras, S.P., Riekkola, M.L. and Kenndler, E., The Principles of Migration and Dispersion in Capillary Zone Electrophoresis in Nonaqueous Solvents, Electrophoresis, Vol.24, No.10(2003), pp.1485-1498.

(39) Muzikar, J., van de Goor, T., Gaš, B. and Kenndler, E., Electrophoretic Mobilities of Large Organic Ions in Nonaqueous Solvents: Determination by Capillary Electrophoresis in Propylene Carbonate, N,N-Dimethylformamide, N,N,-Dimethylacetamide, Acetonitrile and Methanol, Electrophoresis, Vol.23, No.3(2002), pp.375-382. 